\title{
CALCULI OF NET STRUCTURES AND SETS ARE SIMILAR
}

\author{
LUDWIK CzAJA ${ }^{1}$
}

\begin{abstract}
Three basic operations on labelled net structures are proposed: synchronised union, synchronised intersection and synchronised difference. The first of them is a version of known parallel composition with synchronised actions identically labelled. The operations work analogously to the ordinary union, intersection and difference on sets. It is shown that the universe of net structures with these operations is a distributive lattice and - if infinite pre/post sets of transitions are allowed - even a Boolean algebra. As a consequence, some representation theorems of this algebra are stated. The primitive objects are atomic net structures containing one transition with at most one pre-place or post-place (but not both). A simple example of a production system constructed by making use of the operations (and its transformations) is given. Some remarks on behavioural properties of compound nets are stated, in particular, how some constructing strategies may help to infer liveness. The latter issue is limited to semantics of place/transition nets without weights on arrows and with unbounded capacity of places and is not extensively investigated, since the main objective is focused on a calculus of net structures.
\end{abstract}

Mathematics Subject Classification. 68Q85.

\section{INTRODUCTION}

The ascertainment expressed in the title of this paper is prompted by the following observation. Any Petri net-like structure is uniquely represented by its set of transitions, if by a transition a pair of sets (or multisets) is understood: a pre-set and post-set of places. If, in such representation, a place occurs in more than one transition, then it is treated as one in the net structure

\footnotetext{
Keywords and phrases. Net structures, synchronised operations, distributive lattice, Boolean algebra, representation theorems.

1 Institute of Informatics, Warsaw University, ul. Banacha 2, 02-097 Warsaw, Poland; lczaja@mimuw . edu.pl
} 
and, consequently, pictured as a place connected by arrows with all such transitions. Representing net structures as sets induces immediately natural way to combine them - just using set theoretical operations. For instance, net structures $\{(\{x\},\{y, z\}),(\{y, z\},\{u\})\}$ and $\{(\{x, y\},\{v\})\}$ when combined by union $(\cup)$ yield $\{(\{x\},\{y, z\}),(\{y, z\},\{u\}),(\{x, y\},\{v\})\}$, pictorially $x \bigcirc$ In this way, however, new branches (arrows) from/to places only are obtained. To obtain new branches from/to transitions, the latter must also be named (labelled), with the provision that no two distinct transitions may be labelled with the same label in a net structure. A combination of such net structures, called their synchronised union, is obtained by uniting pre-sets (post-sets) of transitions labelled identically in the constituents and leaving transitions labelled differently unchanged. Accordingly, other operations on sets are adapted to net structures. Various operations on nets have been treated since a long time in a number of works, for instance in $[2,3,5,8,11-14,19-21]$. In some of them, like $[8,13,14,21]$, the category theoretic terms have been exploited to this end. However, the main objective of the latter works was a formal description of nets' behaviour, while the present paper aims rather at showing a close affinity of the proposed calculus of net structures and set calculus. Synchronised union, intersection and difference enjoy properties of respective operations on sets, hence a distributive lattice of the net structures and - if infinite pre/post sets of transitions are allowed a Boolean algebra. Thus, some representation theorems hold for them (in [7] a complete lattice of branching processes evoked by a given Petri net has been constructed). Nonetheless, some remarks on how behaviour of a compound net depends on behaviour of its components are stated. Section 2 contains definitions of net structures, labelling of transitions, synchronised union, intersection and difference on net structures and their renaming operation. Some properties of these concepts are stated. In Section 3 the main property of the devised calculus is stated: it is a distributed lattice in any case and a Boolean algebra provided that cardinality of pre and post sets of transitions are not required to be finite. The calculus is illustrated by example of three factories showing how the calculus of net structures may be applied to combine small parts into a large system. Also some remarks on behavioural properties of nets are made. Section 4 contains three isomorphisms of the lattice of net structures, the last of them being an application of the Stone's representation theorem [18] to the calculus of net structures. In Section 5 some final remarks are made.

\section{LABELLED NET STRUCTURES AND THEIR COMPOSITIONS}

\subsection{LABELLED NET STRUCTURE}

Let $\mathbb{X}$ be a set $-\mathrm{a}$ universe of net places for all nets. An unlabelled transition over $\mathbb{X}$ is a pair $t=\left(\bullet t, t^{\bullet}\right)$ where $\bullet^{\bullet} t, t^{\bullet} \subseteq \mathbb{X}$. The set $\bullet^{\bullet}$ is a pre-set and $t^{\bullet}$ a post-set of $t$. An unlabelled net structure over $\mathbb{X}$ is any set $T$ of such transitions. 


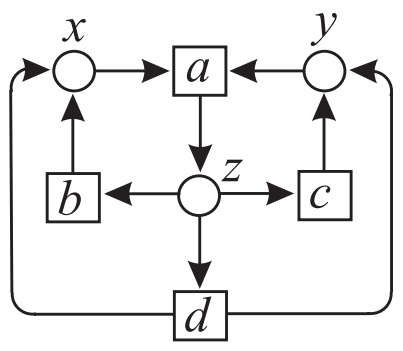

FiguRE 1.

Let members of $T$ be labelled: $l_{T}: L_{T} \stackrel{\text { onto }}{\longrightarrow} T$ is a labelling function, where $L_{T}$ is a set of labels chosen for $T$ so that $\mathbb{X} \cap L_{T}=\emptyset$. Say then " $a$ labels $t$ in $T$ " if $l_{T}(a)=t$. Thus, such a labelled transition is an indexed pair $(a, t)_{T}$ denoted a:t when it is clear from the context which $T$ is involved. Any labelled transition of the form $a:(\emptyset, \emptyset)$ is called isolated. A triple $\left\langle L_{T}, T, l_{T}\right\rangle$ is a labelled net structure, which we often call for short just net structure and denote it also by $T$. This is, thus, a set of labelled transitions in which any label occurs once. The net structure $T=\{a:(\{x, y\},\{z\}), b:(\{z\},\{x\}), c:(\{z\},\{y\}), d:(\{z\},\{x, y\})\}$ is drawn in Figure 1. Here, $l_{T}(a)=(\{x, y\},\{z\}), l_{T}(b)=(\{z\},\{x\}), l_{T}(c)=(\{z\},\{y\})$, $l_{T}(d)=(\{z\},\{x, y\})$. Due to the labelling function and to the implication $a \neq$ $b \Rightarrow a: t \neq b: t$, any ordinary Petri net structure may be represented as a set of labelled transitions. Also the so called non-simple nets, i.e. those where some distinct nodes (places or transitions) have identical pre and post sets, e.g. $a z$ is a set $\{a:(\{x, y\},\{z\}), b:(\{x, y\},\{z\})\}$. Since isolated transitions have no effect on nets' behaviour seen as evolution of state (a part of which, responsible for control, is marking), it is convenient to admit:

Assumption 2.1. Net structures $T$ and $T^{\prime}$ are equal $\left(T=T^{\prime}\right)$ if they differ at most by isolated transitions, i.e. $\{a:(\emptyset, \emptyset)\} \cup T=T$, for any label $a$.

Remark. The manner of labelling admitted here follows that in programming languages, where a statement may be labelled by more than one label, while no two statements in different sites in a program may be labelled by the same label (cf. also TCSP - [9]). Petri net transition is seen as an operator on state, thus a counterpart of statement, and is a unique member of the set $T$. For our purpose such operator may be decorated by more than one label.

Introducing weights on arrows is straightforward: it requires taking multisets instead of sets.

\subsection{Synchronised Union $(\oplus)$ OF NET StRUCTURES}

Since any net structure is a collection of labelled transitions, the operations on sets may be applied to them, but with a restriction: no two different transitions 
with the same label may appear in the resulting net structure (because labelling is a function!). For instance, the net structure $T$ in Figure 1 is a union: $T=\{a$ : $(\{x, y\},\{z\}), b:(\{z\},\{x\}), c:(\{z\},\{y\})\} \cup\{d:(\{z\},\{x, y\})\}$. Such partial union of net structures is capable of generating each net structure from "atoms" if by the latter we mean net structures containing one transition with arbitrary pre/postset. However we need a total operation as well as more elementary "atoms", those of the form $\{a:(\{s\}, \emptyset)\}$ or $\{a:(\emptyset,\{s\})\}$ or $\{a:(\emptyset, \emptyset)\}$ with $s \in \mathbb{X}$ and a label $a$. To obtain arbitrary net structure from such atoms, we define operation called here a synchronised union denoted by $\oplus$. It is defined as follows: for the labelled net structures $T_{1}, T_{2}: \quad \tau \in T_{1} \oplus T_{2}$ iff either there exist labelled transitions $\tau_{i}=a: t_{i} \in T_{i}(i=1,2)$ with $\tau=a:\left(t_{1} \cup t_{2}, t_{1}^{\bullet} \cup t_{2}^{\bullet}\right)$, or $\tau \in T_{1} \cup T_{2}$ otherwise. Due to Assumption 2.1: $\{a:(\emptyset, \emptyset)\} \oplus T=T \oplus\{a:(\emptyset, \emptyset)\}=T$, thus any isolated transition plays a part of the neutral (zero) element for $\oplus$ and will be denoted by $\theta$. Operation $\oplus$ is total, i.e. never two different transitions with the same label appear in the result. For instance, the net structure in Figure 1 is a synchronised union:

$$
\begin{aligned}
T= & \{a:(\{x\}, \emptyset)\} \oplus\{a:(\{y\}, \emptyset)\} \oplus\{a:(\emptyset,\{z\})\} \oplus\{b:(\{z\}, \emptyset)\} \oplus\{b:(\emptyset,\{x\})\} \oplus \\
& \{c:(\{z\}, \emptyset)\} \oplus\{c:(\emptyset,\{x\})\} \oplus\{d:(\{z\}, \emptyset)\} \oplus\{d:(\emptyset,\{x\})\} \oplus\{d:(\emptyset,\{y\})\} .
\end{aligned}
$$

In fact this representation of $T$ is its $\oplus$-decomposition into atomic net structures (associativity of $\oplus$ allows for this notation). The above definitions imply:

\section{Proposition 2.2.}

(i) Synchronised union $\oplus$ of net structures is associative, commutative, idempotent and monotone with respect to relation $\sqsubseteq$ defined as $T_{1} \sqsubseteq T_{2} \Leftrightarrow$ $T_{2}=T_{1} \oplus T_{2}$ being a partial order. The least net structure is $\theta$.

(ii) $T_{1} \oplus T_{2}=\operatorname{lub}\left(T_{1}, T_{2}\right)$ - the least upper bound (wrt $\sqsubseteq$ ) of the set $\left\{T_{1}, T_{2}\right\}$. The operation is total, i.e. $T_{1} \oplus T_{2}$ is always a net structure.

(iii) If $L_{T_{1}} \cap L_{T_{2}}=\emptyset$ then $T_{1} \oplus T_{2}=T_{1} \cup T_{2}$.

(iv) Each net structure is $\oplus$-decomposable into atomic net structures, i.e. containing a single transition with at most one place in its pre-set and then no place in its post-set or, symmetrically, the other way round.

Let atoms $[T]$ denote the set of atoms of $T$. Then $T=\operatorname{lub}($ atoms $[T])$, $T_{1} \oplus T_{2}=\operatorname{lub}\left(\right.$ atoms $\left[T_{1}\right] \cup$ atoms $\left.\left[T_{2}\right]\right)$.

Proof. Evident.

Synchronised union allows to build large nets from small components. It is a version of a parallel composition "||" on nets with synchronised transitions studied e.g. in [12]. However " $\oplus$ " fuses together not only transitions but also places, which is not the case with "\|". In this respect " $\oplus$ " more resembles an operator on nets studied e.g. in [1].

Example 2.3. complete net structures. A net structure $T$ is complete iff each place $s$ has a complement in $T$ i.e. a place $\bar{s}$ such that ${ }^{\bullet} s=\bar{s}^{\bullet}$ and $s^{\bullet}=\boldsymbol{\bullet}^{\boldsymbol{s}}$, where $\bullet^{\bullet} s=\left\{t \in T: s \in t^{\bullet}\right\}$ and $s^{\bullet}=\left\{t \in T: s \in \bullet^{\bullet} t\right\}$. A complement 
of $T$ is a net structure $\bar{T}$ obtained from $T$ by replacing pre-set (post-set) of each transition by its post-set (pre-set) with complemented places (do not mix this notion of complement with that in the lattice theory, where it complements to the greatest element of a lattice!). For any net-structure $T$ the net-structure $T \oplus \bar{T}$, called a complementation of $T$, is complete. For instance, if $T$ is the net-structure in Figure 1 then

$$
\begin{aligned}
\bar{T}= & \{a:(\{\bar{z}\},\{\bar{x}, \bar{y}\}), b:(\{\bar{x}\},\{\bar{z}\}), c:(\{\bar{y}\},\{\bar{z}\}), d:(\{\bar{x}, \bar{y}\},\{\bar{z}\})\} \text { and } \\
T \oplus \bar{T}=\{a:(\{x, y, \bar{z}\},\{z, \bar{x}, \bar{y}\}), b:(\{z, \bar{x}\},\{x, \bar{z}\}), c:(\{z, \bar{y}\},\{y, \bar{z}\}), & d:(\{z, \bar{x}, \bar{y}\},\{x, y, \bar{z}\})\}
\end{aligned}
$$

A more extensive use of the synchronised union is in Example 3.3.

\subsection{SynChronised intersection $(\odot)$ OF NET StruCtures}

Like in case of union, we strengthen ordinary intersection of sets to make it adequate operation on labelled net structures.

First, denote $S U B[T]=\left\{T^{\prime}: T^{\prime} \sqsubseteq T\right\}$, i.e. $S U B[T]$ is a set of all sub-net structures of a net structure $T$, and for a set $A$ of net structures denote by $\operatorname{lub}(A)$ the least upper bound (wrt $\sqsubseteq$ ) of $A$ (if it exists). Obviously, $\operatorname{lub}(S U B[T])=T$. Note that for any sets $A, B$ of net structures: $\operatorname{lu} b(A \cup B)=l u b(A) \oplus \operatorname{lub}(B)$ provided that the $l u b$ s involved exist. Indeed, since $\operatorname{lub}(A), \operatorname{lub}(B) \sqsubseteq \operatorname{lub}(A \cup B)$ then by monotonicity of $\oplus: \operatorname{lub}(A) \oplus \operatorname{lub}(B) \sqsubseteq \operatorname{lub}(A \cup B)$. Conversely, $T \in A \cup B \Leftrightarrow T \in A \vee T \in$ $B \Rightarrow T \in S U B[l u b(A)] \vee T \in S U B[\operatorname{lub}(B)] \Leftrightarrow T \in S U B[\operatorname{lub}(A)] \cup S U B[\operatorname{lub}(B)] \Rightarrow$ $T \in S U B[l u b(A) \oplus \operatorname{lub}(B)]$ (because $S U B\left[T_{1}\right] \cup S U B\left[T_{2}\right] \subseteq S U B\left[T_{1} \oplus T_{2}\right]$ for any net structures $\left.T_{1}, T_{2}\right)$. Hence, $T \sqsubseteq \operatorname{lub}(A) \oplus \operatorname{lub}(B)$. By definition of the $\operatorname{lub}, \operatorname{lub}(A \cup B)$ is the least of all $T^{\prime}$ such that $T \sqsubseteq T^{\prime}$ thus $\operatorname{lub}(A \cup B) \sqsubseteq$ $\operatorname{lub}(A) \oplus \operatorname{lub}(B)$. Therefore $\operatorname{lub}(A \cup B)=\operatorname{lub}(A) \oplus \operatorname{lub}(B)$. From this equality, we get $\operatorname{lub}\left(S U B\left[T_{1}\right] \cup S U B\left[T_{2}\right]\right)=\operatorname{lub}\left(S U B\left[T_{1}\right]\right) \oplus \operatorname{lub}\left(S U B\left[T_{2}\right]\right)=T_{1} \oplus T_{2}$.

Second, by duality, let us define an operation $\odot$ on net structures as $T_{1} \odot T_{2}=$ $\operatorname{lub}\left(S U B\left[T_{1}\right] \cap S U B\left[T_{2}\right]\right)$ called a synchronised intersection of $T_{1}$ and $T_{2}$. Assume $\operatorname{lub}(\emptyset)=\theta$.

\section{Proposition 2.4.}

(i) Synchronised intersection $\odot$ of net structures is associative, commutative, idempotent and monotone with respect to relation $\sqsubseteq$ defined as in Proposition 2.2 and $\theta \odot T=T \odot \theta=\theta$.

(ii) $T_{1} \odot T_{2}=\operatorname{glb}\left(T_{1}, T_{2}\right)$ - the greatest lower bound (wrt $\sqsubseteq$ ) of the set $\left\{T_{1}, T_{2}\right\}$. The operation is total, i.e. $T_{1} \odot T_{2}$ is always a net structure.

(iii) $T_{1} \odot T_{2}=T_{1} \Leftrightarrow T_{1} \oplus T_{2}=T_{2} \Leftrightarrow T_{1} \sqsubseteq T_{2}$.

(iv) $T \odot \bar{T}=\theta$, where $\bar{T}$ is the complement of $T$ (see Ex. 2.3).

(v) $T_{1} \odot T_{2}=\operatorname{lub}\left(\right.$ atoms $\left[T_{1}\right] \cap$ atoms $\left.\left[T_{2}\right]\right)$ (see Prop. 2.2(iv)). 
Proof. Point (i) - evident, (ii) is demonstrated as follows. Let $A=S U B\left[T_{1}\right] \cap$ $S U B\left[T_{2}\right]$. Since $A \subseteq S U B\left[T_{1}\right]$ and $A \subseteq S U B\left[T_{2}\right]$ then, obviously, $T_{1} \odot T_{2}=$ $\operatorname{lub}(A)$ exists in $S U B\left[T_{1}\right]$ and in $S U B\left[T_{2}\right]$, thus in $S U B\left[T_{1}\right] \cap S U B\left[T_{2}\right]$. Therefore $\operatorname{lub}(A) \sqsubseteq T_{1} \wedge l u b(A) \sqsubseteq T_{2}$ and for each $T \in A: \operatorname{lub}(A) \sqsubseteq T \Rightarrow \operatorname{lub}(A)=T$ (because $\operatorname{lub}(A)$, is a maximal element in $A)$. Therefore $\left(T \sqsubseteq T_{1} \wedge T \sqsubseteq T_{2}\right) \Rightarrow T \sqsubseteq l u b(A)$, which means $\operatorname{lub}(A)=g l b\left(T_{1}, T_{2}\right)$. Points $(i i i),(i v),(v)$ - evident.

Synchronised intersection allows to extract some fragments of net structures or to highlight synchronised part of a compound net structure. One may see some affinity of this operator to selection operator in relational data bases: it selects a set of transitions (i.e. a net structure) from one operand under requirements provided in the other.

Example 2.5. For $T$ in Figure 1 and

$$
\begin{aligned}
U & =\{a:(\{x, u\},\{z\}), b:(\{z\},\{x\}), e:(\{z\},\{u\}), f:(\{z\},\{x, u\})\}, \\
T \odot U & =\{a:(\{x\},\{z\}), b:(\{z\},\{x\})\} .
\end{aligned}
$$

A more extensive use of the synchronised intersection is in Example 3.3.

\subsection{Synchronised Difference $(\ominus)$ of net Structures}

By analogy to properties (iv) in Proposition 2.2 and $(v)$ in Proposition 2.4 define a synchronised difference between $T_{1}$ and $T_{2}$ as $T_{1} \ominus T_{2}=\operatorname{lub}\left(\right.$ atoms $\left[T_{1}\right] \backslash$ atoms $\left.\left[T_{2}\right]\right)$. Let us limit ourselves to an analogue to the De Morgan laws in the calculus of sets:

Proposition 2.6. For any net structures $T_{1}, T_{2}, T_{3}$ :

(i) $T_{1} \ominus\left(T_{2} \odot T_{3}\right)=\left(T_{1} \ominus T_{2}\right) \oplus\left(T_{1} \ominus T_{3}\right)$;

(ii) $T_{1} \ominus\left(T_{2} \oplus T_{3}\right)=\left(T_{1} \ominus T_{2}\right) \odot\left(T_{1} \ominus T_{3}\right)$.

Proof. Easy calculation.

Remark. In general $T_{1} \ominus T_{2} \neq \operatorname{lub}\left(S U B\left[T_{1}\right] \backslash S U B\left[T_{2}\right]\right)$.

Synchronised difference allows to delete some fragments of net structures.

Example 2.7. For $T$ in Figure 1 and $U$ in Example 2.5

$$
\begin{aligned}
T \ominus U= & \{a:(\{y\}, \emptyset), b:(\emptyset, \emptyset), c:(\{z\},\{y\}), d:(\{z\},\{x, y\})\}= \\
& \{a:(\{y\}, \emptyset), c:(\{z\},\{y\}), d:(\{z\},\{x, y\})\} \quad \text { (see Assumption 2.1). }
\end{aligned}
$$

A more extensive use of the synchronised difference is in Example 3.3.

\subsection{Renaming of Place names and transition labels}

So far we have defined three set theoretic-like operations on net structures. Now, the calculus will be supplied with the operation of renaming in the style of [15]. 


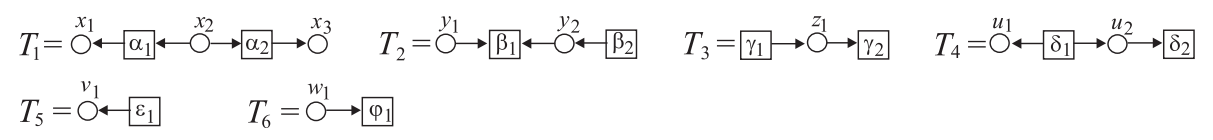

TABLE 1. Renaming functions.

It is a convenient tool for net design, since fragments of a system can be commited to different designers who work separately (a "supervisor" takes their results and makes suitable renaming in order to put them together using operations $\oplus, \odot, \ominus$ ). Let $X_{T} \subseteq \mathbb{X}, L_{T} \subseteq \mathbb{L}$ be sets of places (their names) and transition labels in a net structure $T$ and let $f: X_{T} \cup L_{T} \rightarrow \mathbb{X} \cup \mathbb{L}$ be a mapping called a renaming, such that $x \neq y \Rightarrow f(x) \neq f(y)$ and $x \in X_{T} \Rightarrow f(x) \in \mathbb{X}, x \in L_{T} \Rightarrow f(x) \in \mathbb{L}$. Then, $T[f]$ denotes $T$ with each $x \in X_{T} \cup L_{T}$ replaced by $f(x) \in X_{T[f]} \cup L_{T[f]}$. For instance, let

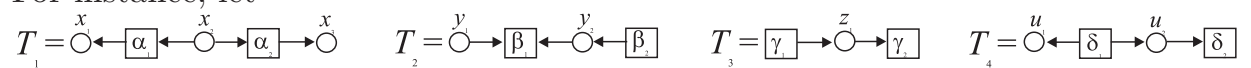
$T=\stackrel{v}{O} \leftarrow \varepsilon \quad T=\stackrel{w}{O} \longrightarrow \varphi_{1}$

and let renaming be given in the Table 1 .

Then the expression $\left(T_{1}\left[f_{T_{1}}\right] \oplus T_{2}\left[f_{T_{2}}\right] \oplus T_{3}\left[f_{T_{3}}\right] \oplus T_{4}\left[f_{T_{4}}\right]\right) \ominus\left(T_{5}\left[f_{T_{5}}\right] \oplus T_{6}\left[f_{T_{6}}\right]\right)$ represents the net structure $T$ in Figure 1.

\section{LATtice OF NET STRUCTURES}

Let $\mathbb{T}$ be the set of all unlabelled transitions over the universe $\mathbb{X}$ of places and let $\mathbb{L}$ be a universe of labels. For $T \subseteq \mathbb{T}$ let $l_{T}: L_{T} \stackrel{\text { onto }}{\longrightarrow} T$ be a labelling function, where $L_{T} \subseteq \mathbb{L}$ is a set of labels in $T$. Additionally assume $L_{\theta}=\emptyset$. Formally, a labelled net structure, not being $\theta$, is the function $l_{T}$, that is a set of all pairs $\left(a, l_{T}(a)\right)$ denoted $a: l_{T}(a)$ with $l_{T}(a) \neq l_{T}(b) \Rightarrow a \neq b$. Let $L N S$ denote the set of all labelled net structures along with $\theta$, that is $L N S=\left\{l_{T} \mid T \subseteq \mathbb{T}\right\}$. For brevity and a part the labelled net structures play here, we retain notation $T$, possibly with indices, for them. It follows from Propositions 2.2 (ii) and 2.4 ( $i i$ ) that $\langle L N S, \oplus, \odot\rangle$ is a lattice.

Moreover we have:

Theorem 3.1. Algebra $\langle L N S, \oplus, \odot\rangle$ is a distributive lattice, i.e. $T_{1} \odot\left(T_{2} \oplus T_{3}\right)=$ $\left(T_{1} \odot T_{2}\right) \oplus\left(T_{1} \odot T_{3}\right)$ and dually, $T_{1} \oplus\left(T_{2} \odot T_{3}\right)=\left(T_{1} \oplus T_{2}\right) \odot\left(T_{1} \oplus T_{3}\right)$ for any net structures $T_{1}, T_{2}, T_{3}$. Its least element is $\theta$. If the universe $\mathbb{X}$ or $\mathbb{L}$ is infinite then assumption $|\bullet t|<\infty$ and $\left|t^{\bullet}\right|<\infty$ for each transition $t \in \mathbb{T}$ deprives the lattice of the greatest element. Without this assumption, the greatest element is the labelled net structure $\mathbb{L} \times\{(\mathbb{X}, \mathbb{X})\}$ thus the lattice is a Boolean algebra. If $\mathbb{X}$ and $\mathbb{L}$ are finite then there are finitely many labelled net structures and the greatest element is their synchronised union (note that in this case $|\bullet t|<\infty$ and $\left|t^{\bullet}\right|<\infty$ hold). For a given $T \in L N S$, the sublattice $\langle S U B[T], \oplus, \odot\rangle$ is a Boolean algebra with the greatest element $T$. 
Proof. First, note that atoms $\left(T_{1} \odot\left(T_{2} \oplus T_{3}\right)\right)=$ atoms $\left(\left(T_{1} \odot T_{2}\right) \oplus\left(T_{1} \odot T_{3}\right)\right)$. Indeed, by easily checked equalities atoms $\left(T{ }_{\odot}^{\oplus} T^{\prime}\right)=\operatorname{atoms}(T) \cup$ atoms $\left(T^{\prime}\right)$ for any $T, T^{\prime}: \operatorname{atoms}\left(T_{1} \odot\left(T_{2} \oplus T_{3}\right)\right)=\operatorname{atoms}\left(T_{1}\right) \cap \operatorname{atoms}\left(T_{2} \oplus T_{3}\right)=\operatorname{atoms}\left(T_{1}\right) \cap$ $\left(\operatorname{atoms}\left(T_{2}\right) \cup \operatorname{atoms}\left(T_{3}\right)\right)=\left(\operatorname{atoms}\left(T_{1}\right) \cap\right.$ atoms $\left.\left(T_{2}\right)\right) \cup\left(\operatorname{atoms}\left(T_{1}\right) \cap\right.$ atoms $\left.\left(T_{3}\right)\right)=$ atoms $\left(T_{1} \odot T_{2}\right) \cup$ atoms $\left(T_{1} \odot T_{3}\right)=$ atoms $\left(\left(T_{1} \odot T_{2}\right) \oplus\left(T_{1} \odot T_{3}\right)\right)$. Hence, lub(atoms $\left.\left(T_{1} \odot\left(T_{2} \oplus T_{3}\right)\right)\right)=\operatorname{lub}\left(\operatorname{atoms}\left(\left(T_{1} \odot T_{2}\right) \oplus\left(T_{1} \odot T_{3}\right)\right)\right) \Leftrightarrow T_{1} \odot\left(T_{2} \oplus T_{3}\right)=$ $\left(T_{1} \odot T_{2}\right) \oplus\left(T_{1} \odot T_{3}\right)$ (see Proposition 2.1 $(i v)$ ). The dual distribution law follows from a general property of distributed lattices.

Second, let $\mathbb{X}=\left\{x_{i} \mid i=1,2,3, \ldots.\right\}, \mathbb{L}=\{a\}$ and $T_{i}=\left\{a:\left(\left\{x_{i}\right\}, \emptyset\right)\right\}$. Then the least upper bound of the set $\left\{T_{1}, T_{2}, T_{3}, \ldots\right\}$ is the labelled net structure $T=\left\{a:\left(\left\{x_{1}, x_{2}, x_{3}, \ldots.\right\}, \emptyset\right)\right\}$ containing one transition with infinite pre-set. The set $L N S_{\text {fin }}$ of all labelled net structures over such $\mathbb{X}$ and $\mathbb{L}$ and containing transitions with finite pre/post sets only, contains $\left\{T_{1}, T_{2}, T_{3}, \ldots\right\}$ as its subset. Thus $L N S_{f i n}$ has no greatest element.

Third, in any case, lub $(L N S)=\mathbb{L} \times\{(\mathbb{X}, \mathbb{X})\}$. Indeed, $(\mathbb{X}, \mathbb{X})$ is the unlabelled transition with the universe $\mathbb{X}$ of places as its pre and post sets. The labelling $l_{\mathbb{T}}$ : $\mathbb{L} \stackrel{\text { onto }}{\longrightarrow} \mathbb{T}$ defined as $l_{\mathbb{T}}(a)=(\mathbb{X}, \mathbb{X})$ is a constant function, thus the set $\{a:(\mathbb{X}, \mathbb{X}) \mid a \in$ $\mathbb{L}\}=\mathbb{L} \times\{(\mathbb{X}, \mathbb{X})\} \in L N S$. Obviously $T \sqsubseteq \mathbb{L} \times\{(\mathbb{X}, \mathbb{X})\}$ and if $\mathbb{L} \times\{(\mathbb{X}, \mathbb{X})\} \sqsubseteq T^{\prime}$ then $T^{\prime}=\mathbb{L} \times\{(\mathbb{X}, \mathbb{X})\}$, for any $T, T^{\prime} \in L N S$.

Fourth, evidently $\operatorname{lub}(S U B[T])=T$, thus $T$ is the greatest element in $S U B[T]$.

Corollary 3.2. Mappings $h_{T_{0}}^{\odot}(T)=T_{0} \odot T$ for any $T_{0}$ are homomorphisms of LNS onto the sublattices of all net structures $T^{\prime}$ with $T^{\prime} \sqsubseteq T_{0}$ and mappings $h_{T_{0}}^{\oplus}(T)=T_{0} \oplus T$ for any $T_{0}$ are homomorphisms of LNS onto the sublattices of all net structures $T^{\prime}$ with $T_{0} \sqsubseteq T^{\prime}$.

Although Theorem 3.1 directly follows from further Theorems 4.1 and 4.2, the latter, as simple versions of representation theorems, have been situated in Section 4 intended for that issue. A general consequence of distributivity of the net structures' lattice is Theorem 4.4. Although it follows from the general Stone's representation theorem [18], it will be proved, since its specificity (as a property of the universe of net structures) makes the proof quite simple.

Example 3.3. Combining production units. There are three production units: $P_{a}, P_{b}, P_{c}$ making aircrafts, boats and cars respectively, and three agencies $S_{a}, S_{b}, S_{c}$ responsible for delivery of the products to a trading company. They are represented by net structures - small loops in Figures 2 and can be combined and optimised in many ways. For instance, separate factories of aircrafts, boats and cars delivering their products at trader's premises (place $s$ ) are represented by net structures $A=P_{a} \oplus S_{a}, B=P_{b} \oplus S_{b}, C=P_{c} \oplus S_{c}$. All the three can be combined into one huge factory $A B C=A \oplus B \oplus C$ represented by a net depicted in Figure 3. Each factory may be optimised by subtracting net structures $T_{\alpha}=\left\{\alpha_{1}:\left(\left\{a_{4}\right\}, \emptyset\right), \alpha_{2}:\left(\emptyset,\left\{a_{4}\right\}\right)\right\}$, $T_{\beta}=\left\{\beta_{1}:\left(\left\{b_{4}\right\}, \emptyset\right), \beta_{2}:\left(\emptyset,\left\{b_{4}\right\}\right)\right\}, T_{\gamma}=\left\{\gamma_{1}:\left(\left\{c_{4}\right\}, \emptyset\right), \gamma_{2}:\left(\emptyset,\left\{c_{4}\right\}\right)\right\}$, depicted in Figure 4. So, $A_{o p t}=A \ominus T_{\alpha}, B_{o p t}=B \ominus T_{\beta}, C_{o p t}=C \ominus T_{\gamma}$. The optimised huge factory is $A B C_{\text {opt }}=A_{\text {opt }} \oplus B_{\text {opt }} \oplus C_{\text {opt }}$, which equals $A B C \ominus\left(T_{\alpha} \oplus T_{\beta} \oplus T_{\gamma}\right)$ and 
by the De Morgan law (Prop. 2.6) also $\left(A B C \ominus T_{\alpha}\right) \odot\left(A B C \ominus T_{\beta}\right) \odot\left(A B C \ominus T_{\gamma}\right)$. It is depicted in Figure 5. If the aircraft and boat factories made an agreement to share the car production then the following units come into being: $A C=A \oplus C$ and $B C=B \oplus C$. Their synchronised intersection is expected to be the car factory. Indeed, $A C \odot B C=(A \odot B) \oplus(A \odot C) \oplus(C \odot B) \oplus(C \odot C)=C$, since $C \odot C=C$ and every remaining summand equals $\theta$. The intended initial marking of all the net structures occurring in this example is the following: one token in $a_{2}, a_{4}, b_{2}, b_{4}, c_{2}, c_{4}$ and no token in remaining places. Note however that the construction of this system and its transformations proceeded on net structures, that is unmarked nets.

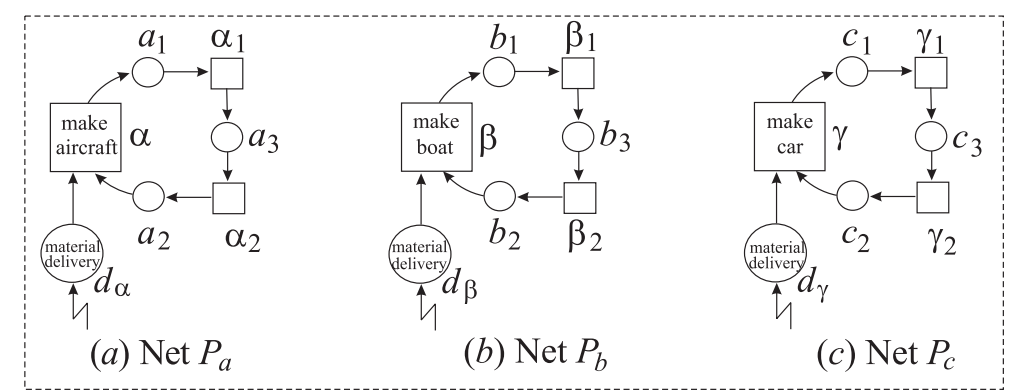

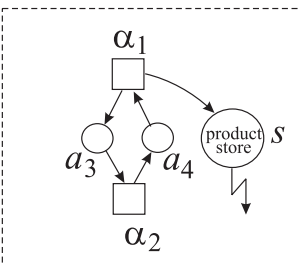

(d) Net $S_{a}$

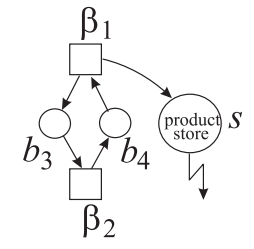

(e) Net $S_{b}$

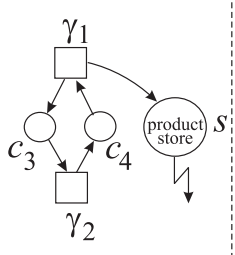

(f) Net $S_{c}$

Figure 2. Production units and delivery agencies.

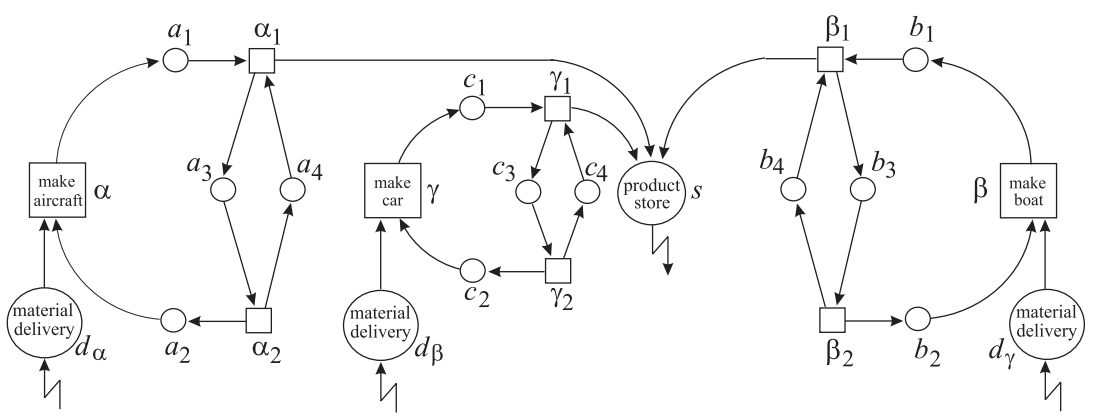

Figure 3. $A B C=\left(P_{a} \oplus S_{a}\right) \oplus\left(P_{b} \oplus S_{b}\right) \oplus\left(P_{c} \oplus S_{c}\right)$. 


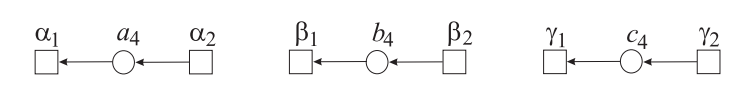

Figure 4. $T_{\alpha}, T_{\beta}, T_{\gamma}$ - substructures to be removed.

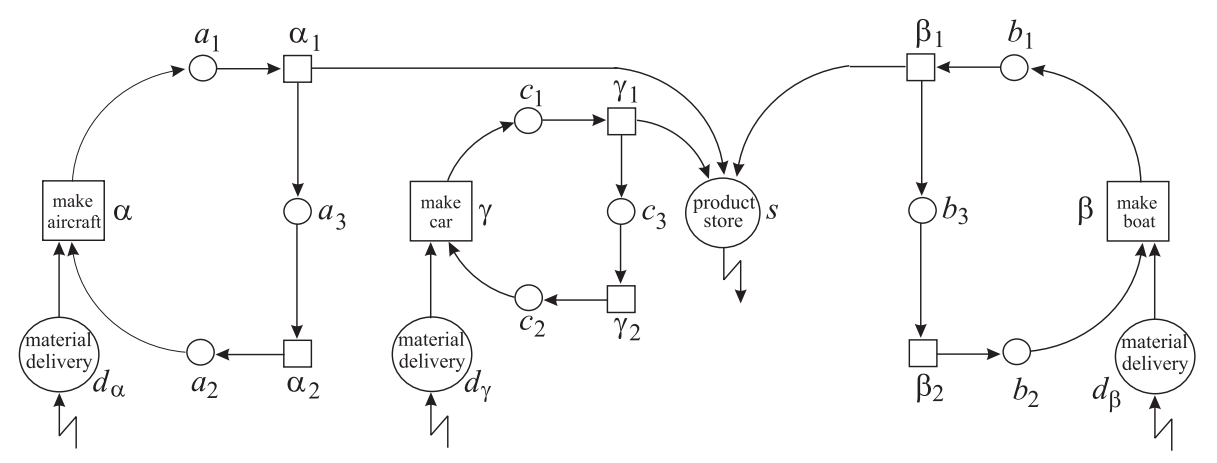

Figure 5. $A B C_{o p t}=A B C \ominus\left(T_{\alpha} \oplus T_{\beta} \oplus T_{\gamma}\right)$.

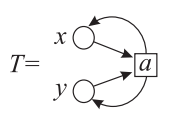

(a)

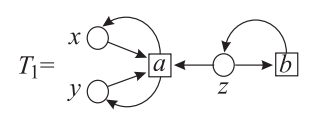

(b)

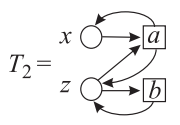

(c)

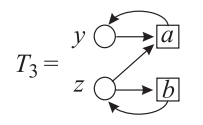

(d)<smiles>COOF</smiles>

(e)<smiles>COBOC</smiles>

(f)

$(e)$

(f)

Figure 6. Parsing trees of net-structure $T$.

Remarks on behavioural properties of compound nets

Remark 3.4. Semantics of nets is a relation between markings. For simplicity, let us define it for Place/Transition nets without weights on arrows and with infinite capacity of places. As above, let $\mathbb{X}$ be a universe of places and $\mathbb{T}$ - the universe of all unlabelled transitions over $\mathbb{X}$. A marking is a function $M: \mathbb{X} \rightarrow \mathbb{N}$ where $\mathbb{N}$ is the set of all natural numbers, 0 including. Note that here the marking does not concern any particular net structure. Markings will be treated as multisets over $\mathbb{X}$ and usual operations,+- and comparison $\leq$ applied component-wise to them. The set $\mathbb{N}^{\mathbb{X}}$ of all markings is denoted by $\mathbb{M}$. For a transition $t \in \mathbb{T}$ by $[[t]]$ is denoted a binary relation between markings, defined by $\left(M, M^{\prime}\right) \in[[t]]$ iff ${ }^{\bullet} t \leq M \wedge M^{\prime}=M-{ }^{\bullet} t+t^{\bullet}$. Semantics of a (unlabelled) net structure $T \subseteq \mathbb{T}$ is a binary relation $[[T]]=\bigcup_{t \in T}[[t]]$. Additionally, $[[\emptyset]]=\emptyset$. The reflexive and transitive closure $[[T]]^{*}$ is a reachability relation in $T$. Note that an ordinary marking of a 
net $T$ is obtained by restricting the marking defined above to the set of places in $T$. If $T$ is labelled (that is $T \in L N S$ ) then its semantics is defined in the same way, since labels do not affect behaviour of a net: we assume $[[a: t]]=[[b: t]]=[[t]]$ for any $a, b \in L_{T}$. Owing to net-independence of marking, we have for any $T_{1}, T_{2} \in L N S$ : If $L_{T_{1}} \cap L_{T_{2}}=\emptyset$ then $\left[\left[T_{1} \oplus T_{2}\right]\right]=\left[\left[T_{1}\right]\right] \cup\left[\left[T_{2}\right]\right]$. Indeed, by (iii) in Proposition $2.2\left[\left[T_{1} \oplus T_{2}\right]\right]=\left[\left[T_{1} \cup T_{2}\right]\right]$. Let $\left(M, M^{\prime}\right) \in\left[\left[T_{1} \cup T_{2}\right]\right]$ thus $\left(M, M^{\prime}\right) \in[[a: t]]$ for a certain labelled transition $a: t \in T_{1} \cup T_{2}$. By $L_{T_{1}} \cap L_{T_{2}}=\emptyset$ : $a$ : $t$ is either in $T_{1}$ or in $T_{2}$ but is not composed of transitions labelled by $a$ in $T_{1}$ and in $T_{2}$. Thus, $\left(M, M^{\prime}\right) \in\left[\left[T_{1}\right]\right] \cup\left[\left[T_{2}\right]\right]$ hence $\left[\left[T_{1} \oplus T_{2}\right]\right] \subseteq\left[\left[T_{1}\right]\right] \cup\left[\left[T_{2}\right]\right]$. Let $\left(M, M^{\prime}\right) \in\left[\left[T_{1}\right]\right] \cup\left[\left[T_{2}\right]\right]$ thus $\left(M, M^{\prime}\right) \in\left[\left[a: t_{1}\right]\right]$ for a certain $a: t_{1} \in T_{1}$ or $\left(M, M^{\prime}\right) \in\left[\left[b: t_{2}\right]\right]$ for a certain $b: t_{2} \in T_{2}$. Thus, $\left(M, M^{\prime}\right) \in\left[\left[T_{1} \cup T_{2}\right]\right]$ hence $\left[\left[T_{1}\right]\right] \cup\left[\left[T_{2}\right]\right] \subseteq\left[\left[T_{1} \oplus T_{2}\right]\right]$.

Remark 3.5. If different net expressions describe the same net structure then they describe different ways of building this net structure. The building process is exhibited by a parsing tree (there is evident grammar of the language of net expressions) of a net expression. Thus, with a given net structure a forest of parsing trees is associated. Some semantic properties of the net may sometimes be inferred from some parsing trees easier than from others. For instance, net structure in Figure 6(a) may be described either as $T_{1} \ominus\left(T_{2} \odot T_{3}\right)$ with the tree in Figure $6(e)$, or (by Prop. 2.6(i)) as $\left(T_{1} \ominus T_{2}\right) \oplus\left(T_{1} \ominus T_{3}\right)$ with the tree in Figure $6(f)$.

Suppose, the net structure $T$ is marked by tokens in $x$ and $y$. If property of liveness is concerned then the tree in Figure $6(f)$ allows to infer liveness of $T$ immediately from liveness of $T_{1} \ominus T_{2}=\{a:(\{y\},\{y\})\}$ and $T_{1} \ominus T_{3}=\{a:(\{x\},\{x\})\}$. Indeed, it is easy to prove that if nets $U, V$ with disjoint sets of places and with markings $M_{U}, M_{V}$ are live then $U \oplus V$ is live in the marking $M_{U} \cup M_{V}$. Note that neither $T_{1}$ nor $T_{3}$ is live.

\section{REPRESENTATION THEOREMS FOR NET STRUCTURES}

The lattice of net structures exhibits a close similarity to the elementary calculus of sets, more exactly, the lattice of subsets of a certain set. We state an isomorphism between the lattice of net structures and three set lattices.

Theorem 4.1. There is one-to-one correspondence between each net structure $T$ and atoms $[T]$ with: atoms $\left[T_{1} \oplus T_{2}\right]=$ atoms $\left[T_{1}\right] \cup$ atoms $\left[T_{2}\right]$ and atoms $\left[T_{1} \odot T_{2}\right]=$ atoms $\left[T_{1}\right] \cap$ atoms $\left[T_{2}\right]$.

In other words, the lattices $\langle L N S, \oplus, \odot\rangle$ and $\langle\{$ atoms $[T]: T \in L N S\}, \cup, \cap\rangle$ are isomorphic.

Proof. If $T_{1} \neq T_{2}$ then obviously atoms $\left[T_{1}\right] \neq \operatorname{atoms}\left[T_{2}\right]$. Let $\operatorname{atoms}\left(T_{1}\right)=$ $\left\{\alpha_{11}, \ldots, \alpha_{1 n}\right\}$, atoms $\left(T_{2}\right)=\left\{\alpha_{21}, \ldots, \alpha_{2 m}\right\}$. Then atoms $\left[T_{1} \oplus T_{2}\right]=$ atoms $[\mathrm{lub}$ $\left(\right.$ atoms $\left[T_{1}\right] \cup$ atoms $\left.\left.\left[T_{2}\right]\right)\right]=$ atoms $\left[\operatorname{lub}\left(\left\{\alpha_{11}, \ldots, \alpha_{1 n}\right\} \cup\left\{\alpha_{21}, \ldots, \alpha_{2 m}\right\}\right)\right]=\left\{\alpha_{11}, \ldots\right.$, $\left.\alpha_{1 n}\right\} \cup\left\{\alpha_{21}, \ldots, \alpha_{2 m}\right\}=$ atoms $\left[T_{1}\right] \cup$ atoms $\left[T_{2}\right]$. For $\odot$ and $\cap-$ similarly. 
Theorem 4.2. There is one-to one correspondence between each net structure $T$ and $S U B[T]$ with: $S U B\left[T_{1} \oplus T_{2}\right]=S U B\left[T_{1}\right] \cup S U B\left[T_{2}\right]$ and $S U B\left[T_{1} \odot T_{2}\right]=$ $S U B\left[T_{1}\right] \cap S U B\left[T_{2}\right]$.

In other words, the lattices $\langle L N S, \oplus, \odot\rangle$ and $\langle\{S U B[T]: T \in L N S\}, \cup, \cap\rangle$ are isomorphic.

Proof. similar to that of Theorem 4.1 - replace atoms with $S U B$.

An important fact in the general theory of lattices is the so called representation theorem originating from [18]. It states that for each distributive lattice there exists an isomorphic set lattice, i.e. a lattice whose members are sets and operations are ordinary union and intersection. Since the lattice of all net structures with synchronised union and intersection is distributive, it has a representation as a set lattice. Let us remind a few facts on ideals - in the context of our lattice - and take a look at such representation proposed in [18]. An ideal of $\langle L N S, \oplus, \odot\rangle$ is any non-empty subset $I \subseteq L N S$ satisfying $(a)\left(T_{1} \in I \wedge T_{2} \in I\right) \Rightarrow T_{1} \oplus T_{2} \in I$, (b) $\left(T_{1} \sqsubseteq T_{2} \wedge T_{2} \in I\right) \Rightarrow T_{1} \in I$ (note that $(a)$ and ( $b$ ) are equivalent to $\left.T_{1} \in I \wedge T_{2} \in I \Leftrightarrow T_{1} \oplus T_{2} \in I\right)$. An ideal $I$ is prime iff $I \neq L N S$ and for any $T_{1}, T_{2}: T_{1} \odot T_{2} \in I \Rightarrow\left(T_{1} \in I \vee T_{2} \in I\right)$. Obviously, if $\neg\left(T_{1} \sqsubseteq T_{2}\right)$ then there exist ideals $I$ such that $T_{1} \in I \wedge T_{2} \notin I$ (example: $\left\{T: T \sqsubseteq T_{1}\right\}$ is such ideal). An ideal $I$ is maximal iff $I \neq L N S$ and for any ideal $I^{\prime} \neq L N S: I \subseteq I^{\prime} \Rightarrow I=I^{\prime}$. In the proof of representation Theorem 4.4, the following fact will be used.

Proposition 4.3. Let $\neg\left(T_{1} \sqsubseteq T_{2}\right)$ and denote by $\mathbb{Q}\left(T_{1}, T_{2}\right)$ the class of all ideals $I$ of the lattice $\langle L N S, \oplus, \odot\rangle$, such that $T_{1} \in I \wedge T_{2} \notin I$. Then $\mathbb{Q}\left(T_{1}, T_{2}\right)$ has a maximal element which, due to distributivity of the lattice, is a prime ideal.

Different versions of this fact are proved (using axiom of choice or its equivalents) in most of expositions of lattice theory, e.g. $[10,16]$.

Theorem 4.4. For a net structure $T \in L N S$ let $\mathbb{P}(T)$ denote the class of all prime ideals $I$ of the lattice $\langle L N S, \oplus, \odot\rangle$, such that $T \notin I$. Then:

(i) $\mathbb{P}\left(T_{1} \oplus T_{2}\right)=\mathbb{P}\left(T_{1}\right) \cup \mathbb{P}\left(T_{2}\right)$ and $\mathbb{P}\left(T_{1} \odot T_{2}\right)=\mathbb{P}\left(T_{1}\right) \cap \mathbb{P}\left(T_{2}\right)$,

(ii) $\mathbb{P}$ establishes one-to-one correspondence between each $T$ and $\mathbb{P}(T)$.

In other words, the lattices $\langle L N S, \oplus, \odot\rangle$ and $\langle\{\mathbb{P}(T): T \in L N S\}, \cup, \cap\rangle$ are isomorphic.

Proof. ( $i) I \in \mathbb{P}\left(T_{1} \oplus T_{2}\right) \Leftrightarrow T_{1} \oplus T_{2} \notin I \Leftrightarrow$ (because $I$ is an ideal) $T_{1} \notin I \vee T_{2} \notin I \Leftrightarrow$ $I \in \mathbb{P}\left(T_{1}\right) \vee I \in \mathbb{P}\left(T_{2}\right) \Leftrightarrow I \in \mathbb{P}\left(T_{1}\right) \cup \mathbb{P}\left(T_{2}\right)$. For $\odot$ and $\cap$ - the dual reasoning.

(ii) If $T_{1} \neq T_{2}$ then $\neg\left(T_{1} \sqsubseteq T_{2} \wedge T_{2} \sqsubseteq T_{1}\right)$ that is $\neg\left(T_{1} \sqsubseteq T_{2}\right) \vee \neg\left(T_{2} \sqsubseteq T_{1}\right)$. Hence, by Proposition 4.1, there exists a prime ideal $I \in \mathbb{Q}\left(T_{1}, T_{2}\right) \cup \mathbb{Q}\left(T_{2}, T_{1}\right)$ that is $T_{1} \in I \wedge T_{2} \notin I$ or $T_{1} \notin I \wedge T_{2} \in I$. Thus, $I \in \mathbb{P}\left(T_{2}\right) \wedge I \notin \mathbb{P}\left(T_{1}\right)$ or $I \in \mathbb{P}\left(T_{1}\right) \wedge I \notin \mathbb{P}\left(T_{2}\right)$, therefore $\mathbb{P}\left(T_{1}\right) \neq \mathbb{P}\left(T_{2}\right)$. 


\section{FINAL REMARKS}

Synchronised union of net structures is an operation appearing (in various guise) in many publications. It is usually called a "parallel combination with synchronisation" and concerns various models of concurrency, not only nets. Contrary to this, synchronised intersection and difference seem to be absent in the theory of parallel processing. This work is rather theoretically oriented and that is why all the operations have been devised to find and study a possibly close correspondence between calculus of net structures and sets. And, possibly, to take advantage of rich substance of the latter, in particular equivalences of net structures like, for instance, an analogue of the De Morgan laws. However, the operations are expected to be of some use in designing large systems from smaller ones and in their transformations. The expectation is supported by a simple example (Ex. 3.3). A particular challenge seems to come from finding conditions making possible to infer some behavioural properties of compound nets from the properties of their components. A modest sample of this issue is hinted in Remark 3.5. This is a subject of current investigations, to be published in a separate article. Besides, combining the idea presented in this paper with equations for message passing worked out in [4] is under investigation.

Acknowledgements. I am really grateful to the anonymous referees who noticed many deficiencies in the first draft of this paper. Especially for making stronger Theorem 3.1 by remark that the constructed calculus is a Boolean algebra (provided that pre/post sets of transitions may assume infinite cardinality), not only a distributive lattice, as stated in the draft. Also for pointing out that exemplary nets in Section 3 do not behave properly in the semantics admitted in the draft.

\section{REFERENCES}

[1] G. Berthelot, Checking properties of nets using transformations, in Advances in Petri Nets, edited by G. Goos and J. Hartmanis. Lect. Notes Comput. Sci. 222 (1985).

[2] E. Best, R. Devillers, M. Koutny, The box algebra = Petri nets + process expressions. Inform. Comput. $\mathbf{1 7 8}$ (2002) 44-100.

[3] L. Czaja, Making Nets Abstract and Structured, in Advances in Petri Nets, edited by G. Goos and J. Hartmanis. Lect. Notes Comput. Sci. 222 (1985) 181-202.

[4] L. Czaja, Equations for message passing. Fund. Inform. 72 (2006) 81-93.

[5] L. Czaja, Interpreted nets. Fund. Inform. 79 (2007) 283-293.

[6] P. Degano, J. Meseguer and U. Montanari, Axiomatising the algebra of net computations and processes. Acta Inform. 33 (1996) 641-667.

[7] J. Engelfriet, Branching processes of Petri nets. Acta Inform. 28 (1991) 575-591.

[8] R. Gorrieri, Refinement, atomicity and transactions for process description languages. Ph.D. Thesis. Dipartimento di Informatica, Universita di Pisa, TD - 2/91 (1991).

[9] C.A.R. Hoare, Notes on Communicating Sequential Processes. Oxford University Computing Laboratory Technical Monograph PRG-33 (1983).

[10] K. Kuratowski and A. Mostowski, Set Theory. North Holland, Amsterdam, PWN, Warsaw (1967). 
[11] A. Mazurkiewicz, Semantics of concurrent systems: a modular fixed point trace approach. Internal Report, Institute of Applied Mathematics and Computer Science, University of Leiden, The Netherlands (1984).

[12] A. Mazurkiewicz, Introduction to Trace Theory, in The Book of Traces, edited by V. Diekert and G. Rozenberg, World Scientific (1995) 3-41.

[13] J. Meseguer and U. Montanari. Petri nets are monoids. Inform. Comput. 88 (1990) 105-155.

[14] J. Meseguer, U. Montanari and V. Sassone, On the Semantics of Place/Transition Petri Nets. Dipartimento di Informatica Universita di Pisa, TR - 27/92 (1992).

[15] R. Milner, Communication and Concurrency. International Series in Computer Science, Prentice Hall (1989).

[16] H. Rasiowa and R. Sikorski, The Mathematics of Metamathematics. PWN, Warsaw (1968).

[17] W. Reisig, Petri Nets, An Introduction. EATCS Monographs on Theoretical Computer Science, Springer Verlag (1985).

[18] M.H. Stone, The theory of representations for Boolean algebras. Trans. Amer. Math. Soc. 40 (1936) 37-111.

[19] H. Wimmel and L. Priese, Algebraic characterisation of Petri net pomset semantics, $C O N$ CUR'97: Concurrency Theory. Lect. Notes Comput. Sci. 1243 (1997) 403-420.

[20] J. Winkowski, An algebraic description of system behaviours. Theoret. Comput. Sci. 21 (1982) 315-340.

[21] G. Winskel, Petri nets, algebras, morphisms and compositionality. Inform. Comput. 72 (1987) 197-238.

Communicated by D. Niwinski.

Received August 3, 2006. Accepted March 2, 2007. 